\title{
Applying the Rhetorical Structure Theory in Alzheimer patients' speech *
}

\author{
Anayeli Paulino and Gerardo Sierra \\ Grupo de Ingeniería Lingüística \\ Instituto de Ingeniería \\ Universidad Nacional Autónoma de México \\ ApaulinoJeiingen . unam.mx \\ GSierraMeiingen.unam.mx
}

\begin{abstract}
In the present paper, semi-structured interviews conducted on 7 Spanish speaking Alzheimer-type Dementia patients and on 6 Spanish speaking adults with healthy aging processes are examined. Rhetorical Structure Theory was used to analyze each of the turns. The procedure involves the segmentation of Semantic Dialog Units (SDU's), rhetorical relations labeling and the construction of tree diagrams. Preliminary results indicate a marked difference in number of rhetoric relations used by both our samples, in which the relations of elaboration, concession, justify and restatement are the most frequently used by Alzheimer-type dementia patients.
\end{abstract}

\section{Introduction}

Dementia of Alzheimer's type (DAT) is a neurodegenerative disease in which at least three cognitive spheres are affected: memory, agnosia and visuospatial skills and language; hitherto, the only definite diagnosis can be determined posmortem.

While linguistic deficits in each phase of dementia have been studied for decades with the intention of identifying the linguistic phenomena which can be significant for a possible diagnosis (Appell, 1982; Kemper, 1991; Emery, 2001), never until recent years has proposal in the field of Natural Language Processing (NLP) been dedicated to utilizing

*This work is supported by CONACYT under the project Ampliación del corpus multiétnico de conversaciones con personas de edad avanzada in collaboration with the Grupo de Ingeniería Lingüística, UNAM, the École de Technologie Supérieure, Montréal and the Universidad Técnica Particular de Loja, Ecuador. the most frequent and useful linguistic deficits, so that, with the help of computational analysis, viable tools in identifying the disease at an early stage can be created.

Thus, recent studies using automated or semiautomated identification have relied on different linguistic criteria such a lexical clues (Bucks et al., 2010; Asgari, Kaye and Dogde, 2017), syntactic complexity (Roark, Mitchel and Hollingshead, 2007), discourse phenomena (Habash, 2011) and even prosodic elements found in narrative language samples (Köning, et al., 2015).

Despite these efforts, there are still limitations as the vast majority of researches have focused solely on English speaking populations. One of the challenges researches have dealt with is using a multiethnic-large- scale Corpus for their studies. Currently Hernández and Ratté (2016) in collaboration with the Carolinas Conversation Collection (Pope and Davis, 2011) are seeking to compile a multiethnic English-Spanish Corpus with the objective to develope an automated tool to detect the most common linguistic deficits in dementia patients.

As an alternative for an innovative analysis that be at the same time useful in the automatic identification of DAT, this paper is based on the Carolinas Conversations Corpus in Spanish and it presents the methodology and first findings on a discourse analysis conducted on Dementia of Alzheimer-type patients using the Rhetorical Structure Theory, RST (Mann and Thompson, 1988). Specifically, the method followed was taken from Maite Taboada's study (2004). The purpose is to identify and extract patterns in the discourse relations (also known 
as rhetorical relations) both in the conversations of Alzheimer-type Dementia patients and normal elderly adults.

\section{Related Work}

Since its proposal, RST has been envisioned as a theoretical model of much utility in the field of Natural Language Processing; evidence of this are the multiple applications in areas as diverse as automatic text summaries, automatic translation, parallel corpus, subjective content analysis and textual similarity among many others.

\subsection{RST for Analysis of Conversations}

The study of spoken discourse has also been the focus of attention of the inquiries concerning RST. Within this field we find the studies by Fawcet and Davies (1992) who propose an analysis of monologue, which can serve as an autonomous discourse unit. Using the original RST proposal by Mann and Thompson, Amanda Stent (2000) offers a new method for segmentation and relations to perform an analysis through turns form which new concepts of relations were created. Moreover, prosodic elements are integrated so as to perform the segmentation.

Maite Taboada (2004) proposes a dialogic analysis method adhering to the original RST. She analyses conversations in Spanish and English from JANUS corpus. Her analysis is contrastive and qualitative intra and inter turns. In her results, she demonstrates that a dialogic analysis can be performed on any language; in addition, she proves that RST is a reliable method to be implemented in spoken discourse.

Other proposals which are not as closely related to RST and which integrate other phenomena have been suggested: DAMSL (Core and Allen, 1997) uses a three layer scheme which integrates the actions of speech along with other filters such as the communicative functions and utterances features. On the other hand, ISO (Bunt et al., 2010) integrates the acts of speech and the classification of emotions for their application in the analysis of dialogs.

\subsection{RST in the Analysis of Dementia}

Up to this point, no study which retakes RST for the discourse analysis on dementia patients has been encountered yet. This notes that whilst RST has had a wide application in areas of computational linguistics, it has not yet been implemented in other interdisciplinary fields such as clinical linguistics.

Notwithstanding this, in the treatment of linguistics deficits, interesting proposals have started to be formulated such as Kong et al. (2017). They used RST in narrations and descriptions of aphasia patients and healthy adults. In the report, it is pointed out that the affected patients produce less discourse units. A breakthrough in this analysis is that the study was strictly discourse-based contrasting the majority of other studies which are based on lexical analysis alone.

\section{Method}

In this section the procedure of this study is described. The criterion for transcription, the labeling of rhetoric relations and the construction and analysis of discourse trees were applied both in injured patients and caregivers. As it was commented before, for the theoretical framework, the analysis proposed by Taboada (2004) was reconsidered for the following reasons: firstly, the author's proposal is the one which most adheres to the standard structure of RST which has the support of a variety of studies; secondly, one of the corpora used was in Spanish, as a consequence the author discusses and takes into account some of the phenomena which are typical of Spanish at the moment of her analysis.

\subsection{Corpus}

In this study, the Carolinas Conversation Corpus in Spanish, which integrates semi-structured interviews conducted on elder adults with Alzheimer disease, elder adults in a healthy aging process, and elder adults with other neurodegenerative diseases was used. The sample consisted of 7 adults diagnosed with Dementia of Alzheimer type in mild and moderate stages ( 6 women, 1 man; average age: $84.57)$, and 6 healthy elderly control participants (6 women; average age: 84). All of the participants lived in Ecuador and were Spanish speakers. Additionally, the alias given by the Carolinas corpus were preserved to protect their privacy. The period between conversations goes from 6 to 50 minutes. 


\begin{tabular}{|c|c|c|c|c|c|c|c|c|c|c|c|c|c|c|}
\hline \multirow{2}{*}{$\begin{array}{l}\text { Relation } \\
\text { Antithesis }\end{array}$} & \multicolumn{2}{|c|}{ Murrieta } & \multicolumn{2}{|c|}{ Cortés } & \multicolumn{2}{|c|}{ San Juan } & \multicolumn{2}{|c|}{ Vicario } & \multicolumn{2}{|c|}{ Mora } & \multicolumn{2}{|c|}{ Zamacona } & \multicolumn{2}{|c|}{ Buendía } \\
\hline & 2 & $3 \%$ & 0 & $0 \%$ & 0 & $0 \%$ & 3 & $7 \%$ & 0 & $0 \%$ & 0 & $0 \%$ & 2 & $4 \%$ \\
\hline Concession & 3 & $5 \%$ & 0 & $0 \%$ & 2 & $11 \%$ & 0 & $0 \%$ & 0 & $0 \%$ & 2 & $14 \%$ & 1 & $2 \%$ \\
\hline Condition & 4 & $7 \%$ & 0 & $0 \%$ & 0 & $0 \%$ & 1 & $2 \%$ & 0 & $0 \%$ & 1 & $7 \%$ & 0 & $0 \%$ \\
\hline Elaboration & 22 & $36 \%$ & 0 & $0 \%$ & 12 & $63 \%$ & 14 & $31 \%$ & 6 & $38 \%$ & 3 & $21 \%$ & 14 & $31 \%$ \\
\hline Evaluation & 2 & $3 \%$ & 0 & $0 \%$ & 2 & $11 \%$ & 1 & $2 \%$ & 0 & $0 \%$ & 0 & $0 \%$ & 0 & $0 \%$ \\
\hline Evidence & 6 & $10 \%$ & 0 & $0 \%$ & 0 & $0 \%$ & 0 & $0 \%$ & 0 & $0 \%$ & 1 & $7 \%$ & 2 & $4 \%$ \\
\hline Justify & 3 & $5 \%$ & 0 & $0 \%$ & 1 & $5 \%$ & 6 & $13 \%$ & 2 & $13 \%$ & 0 & $0 \%$ & 3 & $7 \%$ \\
\hline Motivation & 1 & $2 \%$ & 1 & $20 \%$ & 0 & $0 \%$ & 0 & $0 \%$ & 0 & $0 \%$ & 0 & $0 \%$ & 1 & $2 \%$ \\
\hline Restatement & 3 & $5 \%$ & 1 & $20 \%$ & 0 & $0 \%$ & 3 & $7 \%$ & 4 & $25 \%$ & 2 & $14 \%$ & 2 & $4 \%$ \\
\hline V Cause & 3 & $5 \%$ & 0 & $0 \%$ & 1 & $5 \%$ & 2 & $4 \%$ & 0 & $0 \%$ & 0 & $0 \%$ & 1 & $2 \%$ \\
\hline V Result & 0 & $0 \%$ & 1 & $20 \%$ & 0 & $0 \%$ & 2 & $4 \%$ & 0 & $0 \%$ & 0 & $0 \%$ & 3 & $7 \%$ \\
\hline
\end{tabular}

Table 1: Most frequent Presentational and Subject Matter Relations formulated by DAT patients

\subsection{Annotation criteria}

The norms of the Carolinas Conversations corpus were used. The procedure involved separation in turns (caregiver-patient), followed by the transcription at an orthographic level. For the orthographic level, the conventional signs for punctuation were used: periods, commas, quotation marks, question and exclamation marks. With regards to the prosodic level, intonation phenomena were included (rising/falling), pitch of the voice and long and short pauses (starting from a second on). Finally, in form of comments, kinesthetic phenomena were noted (gestures, sighs, movements), as well as noises and idiolect phenomena for each participant (for instance, use of contractions and diminutives).

For the transcription, the computer program Transcriber 1.5.1 (Boudahmane et al., 2008) was used; the files were converted into txt format so that they could be handled better.

\subsection{Segmentation criteria}

In the initial RST proposal, the minimum units are defined as EDU's (Elementary Discourse Units); they refer to clauses which express a complete meaning (Mann and Thompson, 1988: 6). From this, it is deduced that the segmentation criteria relies on semantic and syntactic limits.

The segmentation units in this study were adapted to the characteristics of the conversational analysis and were denominated as SDU's (Semantic Dialogue Units) (Maite Taboada, 2004: 44). Basically, SDU's are utterances delimited by prosodic features (intonation, pauses), syntactic forms (presence of conjugated verb forms, complements and discourse markers) and semantic criteria (semantic completeness). Semantic units can be compared to previously coined concepts in the field of discourse as "information units" (Halliday, 1967) or "intonation units" (Chafe, 1994). These segmentation criteria are also considered in the analysis by Kong et al. (2017).

An important aspect to keep in mind during the segmentation is the quantity of anomalies presented by patients. The reformulations, the incomplete statements and the unusually long pauses were relatively frequent, therefore, there were some concessions made. For example, syntactic criteria were established as a more reliable limit; however, if there were confusions, the pitch or the intonation disambiguated the boundaries between units. Similarly, on multiple occasions the patient was unable to complete a semantic unit. For these cases, a statement was considered a SDU if it included a conjugated verb and if it was relatively understandable.

\subsection{Labelling}

The rhetoric relations, also known as a coherence or discourse relations (Taboada and Mann, 2006), connect each SDU through functional and semantic features (Mann and Thompson: 5) and the criterion of nuclearity between two semantic units.

During the labeling, it was decided to adhere to the standard classification due to the fact that it is one of the most commonly used classifications; furthermore, unlike others, the number of relations does not hinder identification. The classification was taken form the official RST web-page. ${ }^{1}$

\footnotetext{
${ }^{1}$ www.sfu.ca/rst/
} 


\begin{tabular}{|c|c|c|c|c|c|c|c|c|c|c|c|c|}
\hline \multirow{2}{*}{$\begin{array}{l}\text { Relation } \\
\text { Antithesis }\end{array}$} & \multicolumn{2}{|c|}{ Zubarán } & \multicolumn{2}{|c|}{ DelCarpio } & \multicolumn{2}{|c|}{ Remedios } & \multicolumn{2}{|c|}{ Luna } & \multicolumn{2}{|c|}{ Allende } & \multicolumn{2}{|c|}{ Restrepo } \\
\hline & 6 & $5 \%$ & 2 & $2 \%$ & 0 & $0 \%$ & 1 & $3 \%$ & 3 & $5 \%$ & 3 & $3 \%$ \\
\hline Background & 2 & $2 \%$ & 4 & $5 \%$ & 4 & $3 \%$ & 1 & $3 \%$ & 2 & $4 \%$ & 3 & $3 \%$ \\
\hline Concession & 9 & $8 \%$ & 2 & $2 \%$ & 4 & $3 \%$ & 5 & $16 \%$ & 5 & $9 \%$ & 6 & $6 \%$ \\
\hline Elaboration & 30 & $27 \%$ & 30 & $35 \%$ & 55 & $40 \%$ & 10 & $32 \%$ & 18 & $32 \%$ & 15 & $16 \%$ \\
\hline Evaluation & 2 & $2 \%$ & 2 & $2 \%$ & 1 & $1 \%$ & 0 & $0 \%$ & 3 & $5 \%$ & 8 & $8 \%$ \\
\hline Evidence & 16 & $15 \%$ & 12 & $14 \%$ & 6 & $4 \%$ & 1 & $3 \%$ & 2 & $4 \%$ & 1 & $1 \%$ \\
\hline Interpretation & 3 & $3 \%$ & 2 & $2 \%$ & 1 & $1 \%$ & 0 & $0 \%$ & 1 & $2 \%$ & 2 & $2 \%$ \\
\hline Justify & 3 & $3 \%$ & 4 & $5 \%$ & 10 & $7 \%$ & 2 & $6 \%$ & 1 & $2 \%$ & 7 & $7 \%$ \\
\hline N-V Result & 3 & $3 \%$ & 0 & $0 \%$ & 1 & $1 \%$ & 0 & $0 \%$ & 4 & $7 \%$ & 5 & $5 \%$ \\
\hline Restatement & 8 & $7 \%$ & 3 & $3 \%$ & 4 & $3 \%$ & 0 & $0 \%$ & 1 & $2 \%$ & 18 & $19 \%$ \\
\hline Summary & 5 & $5 \%$ & 4 & $5 \%$ & 3 & $2 \%$ & 0 & $0 \%$ & 0 & $0 \%$ & 1 & $1 \%$ \\
\hline V Cause & 0 & $0 \%$ & 5 & $6 \%$ & 6 & $4 \%$ & 0 & $0 \%$ & 2 & $4 \%$ & 3 & $3 \%$ \\
\hline V Result & 2 & $2 \%$ & 8 & $9 \%$ & 7 & $5 \%$ & 2 & $6 \%$ & 1 & $2 \%$ & 4 & $4 \%$ \\
\hline
\end{tabular}

Table 2: Most frequent Presentational and Subject Matter Relations formulated by healthy elderly controls

\subsection{Discursive-trees elaboration}

For the building of discursive-trees, the RSTTool program (O' Donell, 2004) was employed. Besides the diagrams, this tool offers a statistical section in which it counts the total EDU's and the number of rhetorical relations used in the conversations.

In this first phase it was only applied an intra-turn analysis. The elaboration of discourse trees follows one of the fundamental principles of the theory: the principle of hierarchy between spans and relation schemas (Taboada and Mann, 2006). According to Taboada (2004), the making of discourse trees implies studying the entire text (in this case the entire turn of the patient), locating the biggest, most important and general segments from the surface, and disintegrating them into smaller units. A second analysis is performed inversely: from small units to general ones. In this work both process were made.

Taking into account that an interlocutor always tries to take advantages of their interventions, a nuclear SDU per se was not enough in some cases. It was decided to consider more nuclear semantic units inside the turn if it was required.

During this stage of analysis, anomalies in discourse were reencountered; on some occasions, mostly in the turn of dementia patients, there were ruptures which cannot be related to the main frame, among which, the segment was not united to the main structure and both were considered two different trees. Afterwards, in the organization of relations, if the segments were re- related one another, then these were united to the principal tree.

\subsection{Preliminary results}

Tables 1 and 2 show most frequent rhetorical relations used by DAT patients and healthy elderly controls, respectively. Until now, the results of the quantitative analysis point out that there are marked differences in the frequency of rhetoric relations produced between the population with dementia and the healthy older controls. Additionally, patients with dementia use less variety of rhetoric relations in their conversational discourse than adults without cerebral damage.

Out of all the rhetorical relations, there is a frequent use of elaboration relation in both populations. Despite the fact that the use of elaboration is more frequent that other forms of relations, particularly in the patients with Alzheimer-type dementia, other relations are recurrent as well; there are concession, justify and restatement. It is particularly remarkable that relations like enablement, unconditional and purpose were not employed by either of the groups.

Based on data, it is observed that, roughly, most used relations in elderly adults were also frequently used by DAT patients, hence, it seems that the main difference between the samples relies on the proportion of relations used.

\subsection{Future Work}

Even though this study employed a limited number of DAT patients, the sample allows the exploration of RST as a reliable method for describing coherence relations established between semantic units in a dialog. However, it is still necessary to carry out 
contrastive studies with other corpus in order to corroborate the results obtained in this work.

Many tasks are yet to be done in the present proposal, one of which is the examination of the tree diagrams of each speaker. The number of nodes and the levels of each scheme will result helpful in doing so. The analysis in between turns is still missing, which will be challenging considering the phenomena involved. Consequently, this makes them difficult to integrate into the general diagram scheme. In spite of the connection which can be established between turns, we must consider necessary restrictions that the discourse genre imposes.

This paper merely examines the discourse of a small sample of Alzheimer-type Dementia patients and healthy adults; nevertheless, the analysis method of RST seems to be viable up until its incipient stage of analysis. For this reason further applications in the field of linguistic deficits are particularly promising.

\section{References}

Alexandra Köning, Aharon Satt, Alexander Sorin, Ron Hoory, Orith T. Ronen, Alexandre Derreumaux, Valeria Manera, Franz Verhey, Pauline Aalten, Pillipe H. Robert and Renaud David. 2015. Automatic speech analysis for the assessment of patients with predementia and Alzheimer's disease. Alzheimer and Dementia, 1 (1): 112-124.

Amanda Stent. 2000. Rhetorical Relations in dialog. INLG 00 Proceedings of the first international conference on Natural Language generation, Mitzpe, Ramón, Israel.

Antony Habash. 2012. Language analysis of speakers with dementia of the Alzheimer's type. University of North Carolina Wilmington.

Anthony Pak-Hin Kong, Anastasia Linnik, Sam-Po Law and Waisa Wai-Man. 2017. Measuring discourse coherence in anomic aphasia using Rhetorical Structure Theory. International Journal of Speech-Language Pathology, 1-16.

Brian Roark, Margaret Mitchel and Kristy Hollingshead. June 2007. Syntactic complexity measure for detecting Mild Cognitive Impairment. BioNLP 2007: Biological, translational, and clinical language processing. Prague: 1-8.

Charlene Pope and Boyd H. Davies. 2011. Finding a balance: The Carolinas Conversation Collection. Corpus Linguistics and Linguistic Theory, 7(1): 143-161.
Daniel Kempler. 1991. Language Changes in Dementia of the Alzheimer Type. Dementia and Communication. Philadelphia, B.C. Decker.

Harry Bunt, Jan Alexandersson, Jae-Woong Choe, Alex Chengyu Fang, Koiti Hasida, Volha Petukhova, Andrei Popescu-Belis, Laurent Romary and David Traum. 2010. ISO 24617-2: A semantically-based standard for dialogue annotation. Proceedings of LREC2012.

Julian Appell, Andrew Kertesz and Michael Fisman. 1982. A Study of Language Functioning in Alzheimer Patients. Brain and Language. 17, 73-91.

Karim Boudahmane, Mathieu Manta, Fabien Antoine, Sylvain Galliano and Claude Barras. 2008. Transcriber: a tool for segmenting, labeling and transcribing speech.

Laura Hernández, Sylvie Ratté, Charlene Pope and Boyd H. Davis. August 11, 2016. Conversing with the elderly in Latin America: a new cohort for multimodal, multilingual longitudinal studies on aging. Proceedings of the 7th Workshop on Cognitive Aspects of Computational Language Learning, 16-21.

Maite Taboada. 2004. Building coherence and cohesion: task-oriented dialogue in English and Spanish. Amsterdam, John Benjamins.

Maite Taboada and William Mann. 2006. Rhetorical Structure Theory: looking back and moving ahead. Discourse Studies, 8(3): 423-459.

M.A.K. Halliday. Notes on transitivity and theme in English. Journal of Linguistics, 3: 177-274.

Mark G. Core and James F. Allen. 1997. Coding Dialogs with DAMSL Annotation Scheme. AAAI fall symposium on communicative action in humans and machines, 56.

Meysam Asgary, Jeffrey Kaye and Hiroko Dodge. 2017. Predicting mild cognitive impairment from spontaneous spoken utterances. Alzheimer's \& Dementia, 3: 219-228.

Mick O’Donell. 2004. RSTTool. An RST Markup Tool.

Olga Emery, 2001. Language impairment in dementia of the Alzheimer type: A hierarchical decline? International Psychiatry in medicine, 30 (2), 145-164.

Robin P. Fawcett and Bethan L. Davies. April, 1992. Monologue as a turn in dialogue: towards an integration of exchange structure and rhetorical structure theory. 6th International Workshop on Natural Language Generation. Trente, Italy.

Wallace Chafe. 1994. Discourse, consciousness, and time, Chicago, The University of Chicago Press.

Willian Mann and Sandra Thompson. 1988. Rhetorical Structure Theory: Towards a functional theory of text organization. Text, 8, 243-281. 